\title{
Post-transplant lymphoproliferative disorder in hematopoietic stem cell transplant patients: A single center retrospective study between 2005 and 2012
}

\author{
JOANA MARINHO-DIAS ${ }^{1-3}$, JOÃO LOBO ${ }^{3-5}$, RUI HENRIQUE ${ }^{3-5}$, INÊS BALDAQUE ${ }^{1}$, \\ CARLOS PINHO-VAZ ${ }^{6}$, LUÍSA REGADAS ${ }^{6}$, ROSA BRANCA ${ }^{6}$, FERNANDO CAMPILHO ${ }^{6}$, \\ ANTÓNIO CAMPOS JR ${ }^{6}$, RUI MEDEIROS ${ }^{1-3,7}$ and HUGO SOUSA ${ }^{1,2}$
}

\begin{abstract}
${ }^{1}$ Virology Service, Portuguese Oncology Institute of Porto; ${ }^{2}$ Molecular Oncology and Viral Pathology Group, IPO-Porto Research Center (CI-IPOP), Portuguese Oncology Institute of Porto, 4200-072 Porto;

${ }^{3}$ Abel Salazar Institute for The Biomedical Sciences (ICBAS), University of Porto, 4050-313 Porto;

${ }^{4}$ Department of Pathology, Portuguese Oncology Institute of Porto; ${ }^{5}$ Cancer Biology and Epigenetics Group, IPO-Porto Research Center (CI-IPOP); ${ }^{6}$ Bone Marrow Transplantation Unit, Portuguese Oncology Institute of Porto, 4200-072 Porto; ${ }^{7}$ Research Department, Portuguese League Against Cancer (LPCC-NRNorte), 4200-172 Porto, Portugal
\end{abstract}

Received November 6, 2017; Accepted April 6, 2018

DOI: $10.3892 / \mathrm{mmr} .2018 .9433$

\begin{abstract}
Post-transplant lymphoproliferative disorder (PTLD), despite its rarity, is an important mortality/morbidity event in transplant patients. The purpose of the present study was to retrospectively examine the clinical and pathologic characteristics, and outcomes of PTLD at the Portuguese Oncology Institute of Porto. A retrospective review of patient information was performed for patients that developed PTLD following allogeneic hematopoietic stem cell transplant (aHSCT) and were diagnosed between 2005 and 2012. The present study included a total of 15 patients, 8 females (53.3\%) and 7 males (46.7\%), with different clinicopathological characteristics. The most frequent clinical condition inducing aHSCT was acute lymphocytic leukemia (40.0\%). Conditioning regimens consisted primarily in busulfan and cyclophosphamide, with anti-thymocyte globulin, and myeloablation was the preferential treatment. Epstein-Barr virus (EBV) was present in all patients with a median time of diagnosis following transplant of 75 days (range, 25-485 days) and a median viral load of $4.75 \log _{10}$ copies/ml (range, 3.30-6.26 $\log _{10}$ copies/ml). PTLD diagnosis was mainly assessed by clinical findings, and histological confirmation was available for 5 patients:
\end{abstract}

Correspondence to: Dr Joana Marinho-Dias or Professor Hugo Sousa, Virology Service, Portuguese Oncology Institute of Porto, Rua Dr António Bernardino Almeida, 4200-072 Porto, Portugal

E-mail: joana_marinho_dias@hotmail.com

E-mail: hugomls@gmail.com

Key words: post-transplant lymphoproliferative disorder, post-transplant lymphoproliferative disease, Epstein-Barr virus
3 monomorphic, 1 polymorphic and 1 with early lesions of PTLD. To the best of our knowledge, this is the first study to describe PTLD cases in HSCT patients in Portugal. The data reinforces the importance of performing EBV monitoring in high-risk patients, particularly those receiving a transplant from mismatch/unrelated donors, and those with myeloablative conditioning regimen including antithymocyte globulin. The results also suggested that EBV viral load may be significant for the prediction of PTLD development.

\section{Introduction}

The development of lymphoid proliferations after transplantation has been recognized for more than a quarter of century as an important morbidity factor (1). The post-transplant lymphoproliferative disorder (PTLD) refers to a heterogeneous group of lymphoproliferative diseases, which vary from uncomplicated, self-limiting infectious mononucleosis, to malignant lymphoma. The histological characterization varies from reactive-appearing, polyclonal lymphoid infiltrates or undifferentiated cells that are morphologically indistinguishable from malignant lymphoma or plasma cell myeloma (2-4).

PTLD is relatively rare; nevertheless, it is the most frequent malignant disease early after transplantation, with the majority of cases being reported in the first year after transplantation $(3,5,6)$. Risk factors for PTLD development include young age and age over 50 years at transplantation, white race, unrelated or HLA-mismatched graft, Epstein-Barr virus (EBV)-seronegative status prior to transplant, primary EBV infection, type of organ transplant, intensity of immunosuppression and the occurrence of concomitant cytomegalovirus disease $(3,7)$.

Not all PTLD cases are EBV-related, but consistent data recognize primary EBV infection as the most important risk factor for PTLD development $(8,9)$. Indeed, the immunosuppression after transplantation in an EBV-seropositive 
patient reduces the activity of the patients' EBV-specific cytotoxic T-cell surveillance, which increases the probability of uncontrolled proliferation of EBV-infected B-cells and subsequent progression to PTLD (10). Moreover, transplant recipients experiencing primary EBV infection during the early post-transplant period seem to be particularly susceptible to develop PTLD of B-cell origin, reflecting their lack of any preexisting EBV-specific T-cell immunity $(3,10)$.

The overall incidence of PTLD varies from 1 to $22 \%$ depending on the presence of risk factors, namely the transplanted organ, patient age, EBV serostatus from recipient and donor, aggressiveness of immunosuppression (11). The cumulative incidence of PTLD in allogeneic hematopoietic stem cell transplantation (aHSCT) recipients is approximately $1.0 \%$ (range $0.5-1.8 \%$ ), with slightly higher rates in the pediatric population $(1,12)$. Survival rates depend mainly on the type of PTLD, extent of disease and patient age: While pediatric patients and those with localized disease seem to have a better prognosis, monomorphic PTLDs are the most aggressive forms $(5,7,13)$.

The purpose of this study was to examine the clinical and pathologic characteristics, as well as the outcome of PTLD after aHSCT, in patients diagnosed at the Portuguese Oncology Institute of Porto (Porto, Portugal) between 2005 and 2012.

\section{Materials and methods}

Type of study and study participants. We retrospectively reviewed the clinic-pathological and EBV infection data of patients that developed PTLD after aHSCT at the Bone Marrow Transplantation Unit from Portuguese Oncology Institute of Porto in 2005 and 2012. This retrospective study was approved by the Ethical Committee of IPO Porto. The present study included a total of 15 patients, 8 females $(53.3 \%)$ and 7 males $(46.7 \%)$. When available, cases were histologically confirmed by an expert pathologist and classified according the most recently available edition of the World Health Organization (WHO) Classification of Tumours of Haematopoietic and Lymphoid Tissues (4th edition).

Sample processing and EBV detection. Samples were collected in EDTA-containing tubes (Vacutainer ${ }^{\circledR}$; BD Biosciences, Franklin Lakes, NJ, USA) and stored in freezing temperature prior to processing. Blood samples were collected retrospectively from the institution archives. DNA was extracted by MagNA Pure Compact Nucleic Acid Isolation kit I (Roche Diagnostics GmbH, Mannheim, Germany). DNA/RNA quality was assessed by measuring the absorbance at 260/280 nm using the NanoDrop 1000 Spectrophotometer v3.7 (Thermo Fisher Scientific, Inc., Wilmington, DE, USA).

All patients submitted to aHSCT were monitored for EBV infection upon request from clinicians after clinical suspicion. EBV detection was performed at the Virology Service of IPO Porto using the commercial Real-Time PCR kit EBV Q-PCR Alert (Nanogen Advanced Diagnostics S.p.A., Trezzano sul Naviglio, Italy) which targets a region from EBV nuclear antigen 1 gene (EBNA1). Amplification was performed with the ABI PRISM 7300 Sequencer Detection System (Applied Biosystems; Thermo Fisher Scientific, Inc., Waltham, MA, USA) and results were obtained by measuring the geometric
Table I. Clinical characteristics of patients.

\begin{tabular}{|c|c|}
\hline Variable & $\mathrm{n}(\%)$ \\
\hline Age, median (range); years & $10(3-38)$ \\
\hline \multicolumn{2}{|l|}{ Sex } \\
\hline Male & $7(46.7)$ \\
\hline Female & $8(53.3)$ \\
\hline \multicolumn{2}{|l|}{ Underlying disease } \\
\hline Acute leukemia & $10(66.6)$ \\
\hline Chronic leukemia & $1(6.7)$ \\
\hline Myelodysplastic/myeloproliferative syndrome & $1(6.7)$ \\
\hline Others & $3(20.0)$ \\
\hline \multicolumn{2}{|l|}{ HLA donor } \\
\hline Match/related & $4(26.7)$ \\
\hline Mismatched/unrelated & $11(73.3)$ \\
\hline \multicolumn{2}{|l|}{ Source of cells } \\
\hline PBSC & $12(80.0)$ \\
\hline $\mathrm{BM}$ & $2(13.3)$ \\
\hline $\mathrm{UCB}$ & $1(6.7)$ \\
\hline \multicolumn{2}{|l|}{ Conditioning regimen } \\
\hline MAC & $14(93.3)$ \\
\hline RIC & $1(6.7)$ \\
\hline \multicolumn{2}{|l|}{ ATG } \\
\hline Yes & $12(85.7)$ \\
\hline No & $2(14.5)$ \\
\hline
\end{tabular}

ATG, anti-thymocyte globulin; PBSC, peripheral blood stem cells; $\mathrm{BM}$, bone marrow; UCB, umbilical cord blood; MAC, myeloablative conditioning; RIC, reduced-intensity conditioning; HLA, human leukocyte antigen.

increase of probe fluorescence during amplification and samples were considered positive when the exponential curve exceeded the cycle threshold line.

Regarding amplification quality, positive and negative controls were used: as negative control we used double distilled water in replacement of template DNA; and as positive control we have used samples from the external quality control panel used at the Virology Service for EBV diagnosis.

Data collection. Clinic-pathological data was extracted from institutional databases including pre-transplant recipient age, gender, underlying disease, HLA-donor-recipient status, EBV serological status of the recipient, source of stem cells, conditioning regimen and use of ATG; post-transplant information (clinical findings, date of PTLD suspicion, date of PTLD confirmation, PTLD type, GVHD prophylaxis, GVHD type and outcome) and viral data (date of EBV suspicion, EBV viral load).

Statistical analysis. Statistical analysis was performed using the SPSS version 20.0 software (IBM Corp., Armonk, NY, USA). Overall survival was defined as the time between the date of transplant and the date of last follow-up or mortality. The differences in survival were calculated using the log-rank test and the Kaplan-Meier method. 


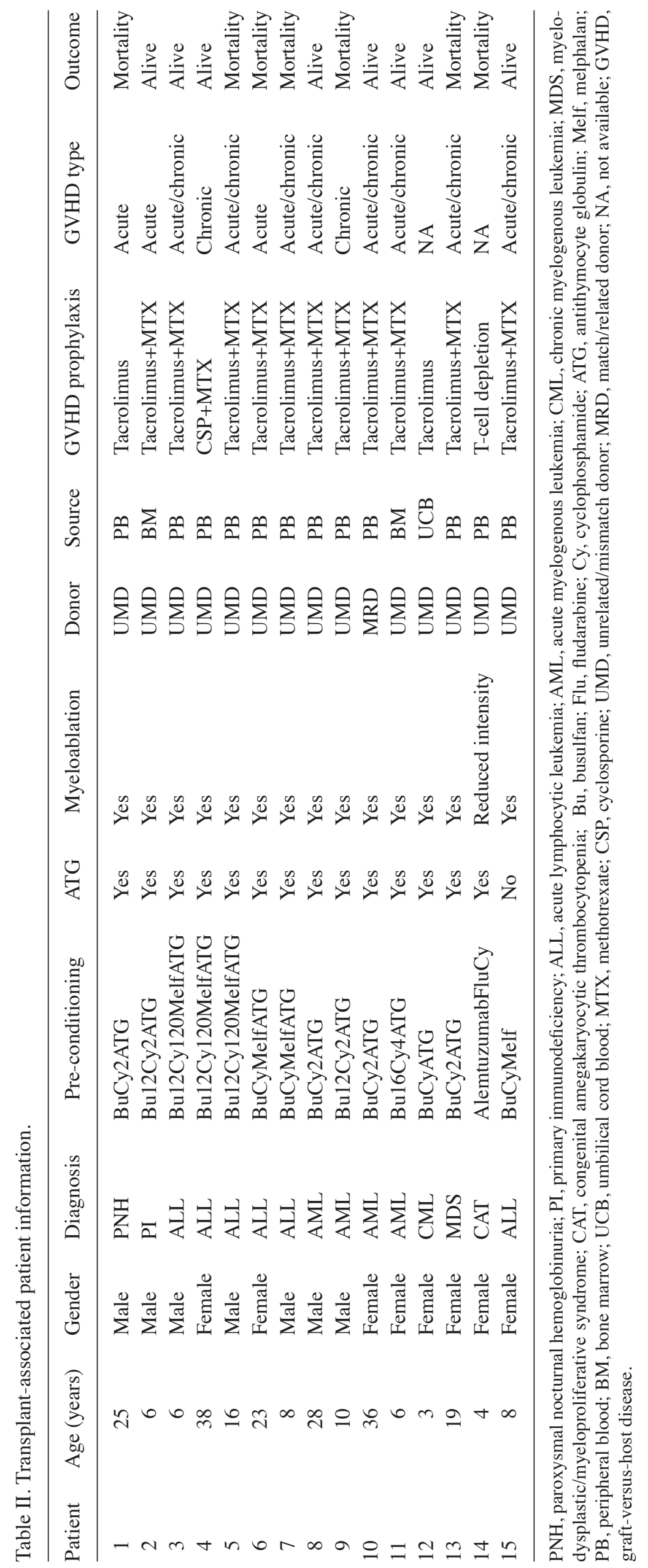




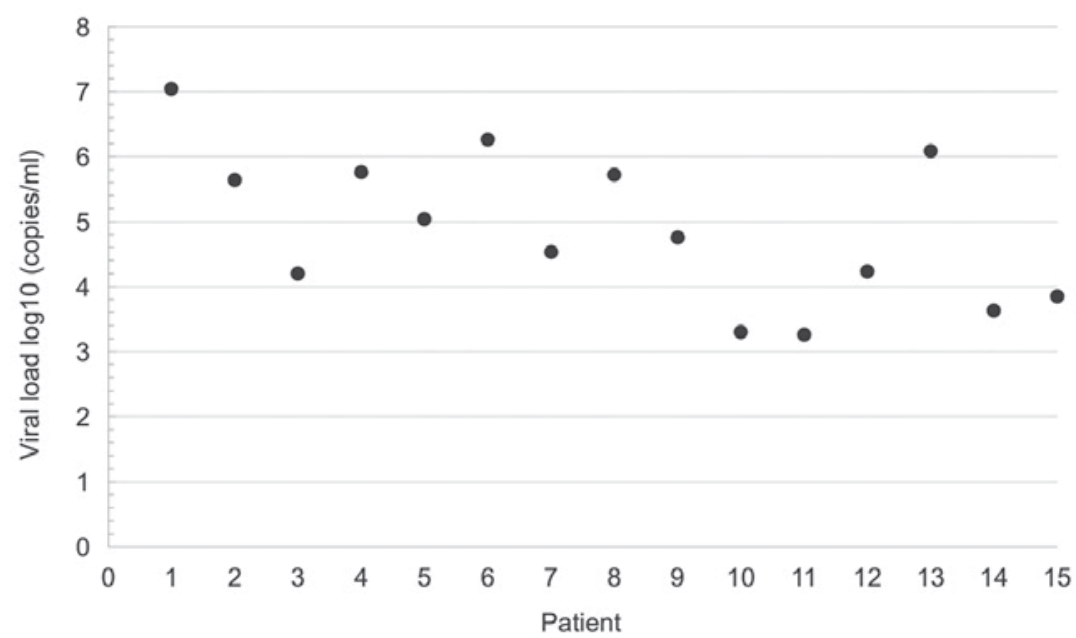

Figure 1. Viral load distribution from all patients involved in the retrospective study.

\section{Results}

The study included a total of 15 patients, 8 females (53.3\%) and 7 males (46.7\%), with median age of 10 years-old (range 3-38)- Table I. Patients had a median follow-up time of 14 months (range: 2-72). Primary diagnoses of patients included in this study included paroxysmal nocturnal hemoglobinuria $(n=1)$, primary immunodeficiency $(n=1)$, acute lymphocytic leukemia $(n=6)$, acute myelogenous leukemia $(n=4)$, chronic myelogenous leukemia $(n=1)$, myelodysplastic/myeloproliferative syndrome $(n=1)$ and congenital amegakaryocytic thrombocytopenia $(\mathrm{n}=1)$. Most of patients had mismatched/unrelated donors (73.3\%) and the collection of cells was mainly performed by peripheral blood stem cells $(80.0 \%)$. Myeloablative conditioning was used in 14 patients and ATG in 12 patients. Transplant-related information for each patient is described in Table II.

Regarding the clinical presentation of patients, 2 presented with fever, 12 had increased liver enzymes, adenomegalies were observed in 2 patients and 12 patients had also increased lactate dehydrogenase. EBV serological status prior to transplantation were evaluated according to presence of IgM and IgG titers in plasma samples. Serological status was divided in three groups: susceptible (absence of IgM and IgG), active infection (presence of IgM and/or IgG) and finally, past infection (absence of $\operatorname{IgM}$ and presence of $\operatorname{IgG}$ ).

The development of EBV infection was present in all of 15 patients, with a median time of diagnosis after transplant of 68 days (range 29-464 days), with $80 \%(n=12)$ of them detected $<180$ days after transplant, and with a median viral load of $4.75 \log _{10}$ copies $/ \mathrm{ml}$ (range 3.30-6.26 $\log _{10}$ copies $/ \mathrm{ml}$; Fig. 1). PTLD diagnosis occurred approximately in the same period where EBV infection occurred (mean 135, median 75 days and range 25-485 days vs. mean 130 days, median 68 days and range 29-464 days, respectively). PTLD classification was available for only 5 patients and included monomorphic-type PTLD $(n=3)$, polymorphic PTLD $(n=1)$ and reactive plasmocytic hyperplasia (early lesions) $(n=1)$ (Table III; Fig. 2). The remaining cases were not histological confirmed, and diagnosis was established by considering all clinical findings.
We observed graft-vs.-host disease (GVHD) in 13 patients (93.3\%): 3 with acute GVHD (20.0\%), 2 with chronic GVHD $(13.3 \%)$ and 8 with both (53.3\%). Considering the grade of acute GVHD, all patients with clinical information had a grade of II or higher. Regarding chronic GVHD, 3 patients had an evolution of acute-to-chronic, while 7 had a de novo chronic GVHD; two patients experienced extensive disease and 5 had only limited disease (Table II).

As for the overall outcome, 8 patients are still alive (53.3\%), 5 with no signs of disease (33.3\%) and 3 with evidence of disease (20.0\%); and 7 patients have died (46.7\%), 4 from complications associated with the transplant (26.7\%) and 3 from progression of disease (20.0\%) (Table II). A Kaplan-Meier plot was obtained by evaluating the cumulative survival of these patients, in months (Fig. 3).

\section{Discussion}

PTLD is one of the most serious complications of immunosuppression in patients who undergo hematopoietic stem cell transplantation, with high impact on morbidity and mortality (14). EBV infection has been strongly associated with the development of PTLD, and this association is widely described in the literature $(15,16)$.

In this retrospective analysis, we verified that PTLD affects individuals of all age groups and with several types of hematological malignancies, the majority having had unrelated donors. Our patients had different types of pre-conditioning regimens (myeloablative in 14 patients), with predominance of busulfan and cyclophosphamide. Since types of regimens are varied, they appear not to have a direct correlation with the development of PTLD. ATG was used in almost all patients except for two, and without absolute prevalence date it is difficult to confirm if its use is directly correlated with PTLD development. GVHD prophylaxis was performed mainly with tacrolimus, and concomitant with MTX, and still patients have developed some type of GVHD which indicates that altering prophylaxis regimen should be taken into consideration.

In our case series, EBV infection was diagnosed at a median of 68 days after transplant. EBV infection is frequently associated with the intermediate period after aHSCT, mainly 
$\frac{.0}{\frac{9}{4}}$

충

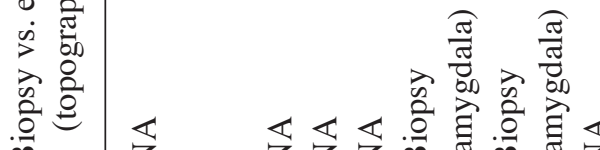

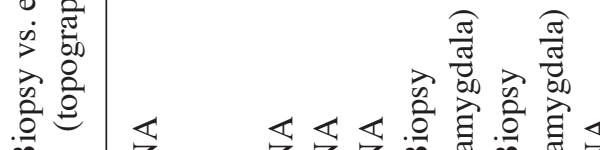

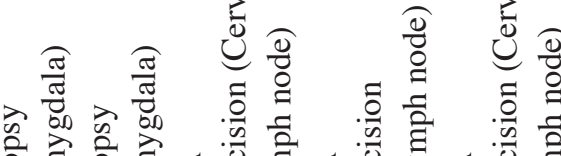

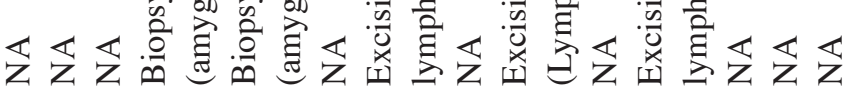

它

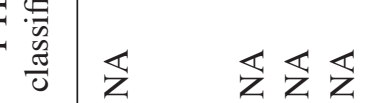

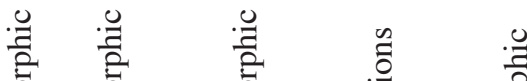

节

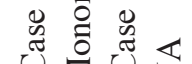

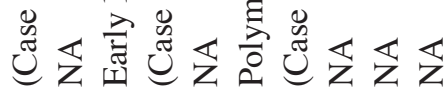

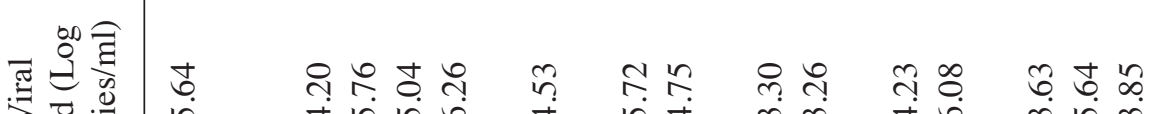

$>$ चु

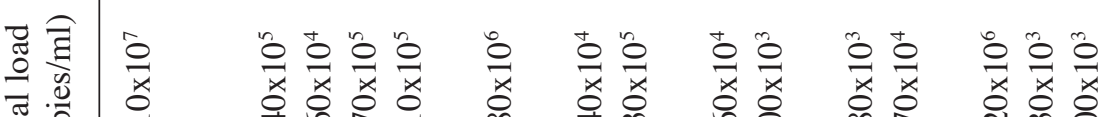

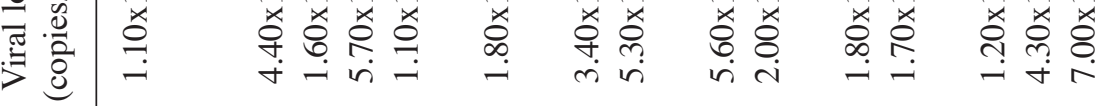

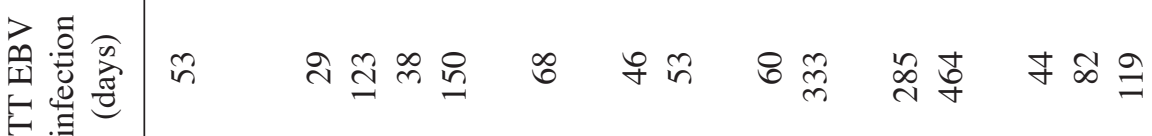

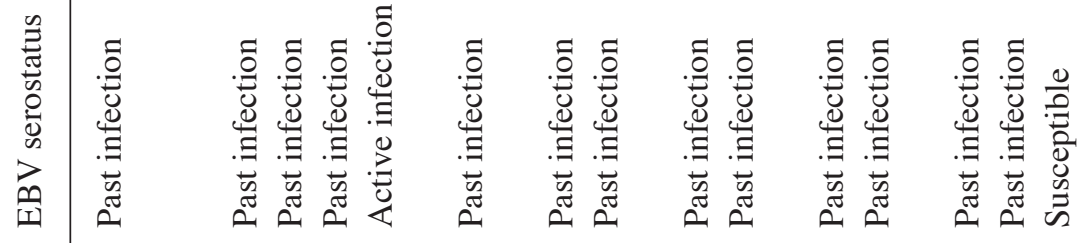

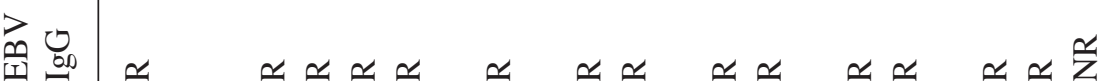

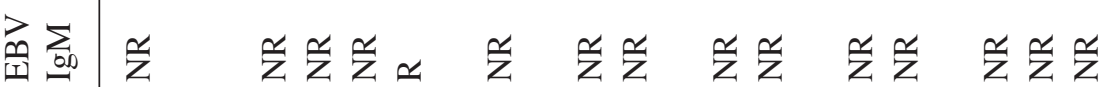

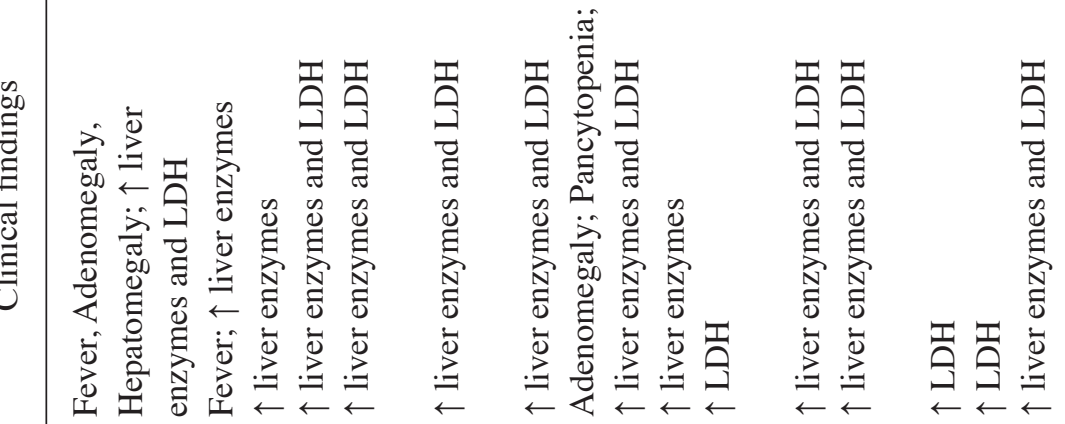

$\frac{n}{6}$

.ึ.

$\underset{\mathrm{I}}{\mathrm{I}}$

모뤈

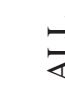

究蓆

$\sum \sum$

$\sum_{<}$

它 $\frac{0}{0}$

$\sum^{\frac{0}{\pi}}$

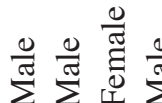

䒕

苟

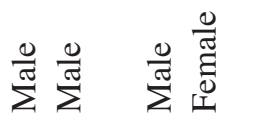

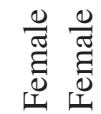

赵离

$\circ 6 \infty$

$\dot{\Xi}$

表

\section{$\ddot{\imath}$}

$\infty \stackrel{\infty}{\sim}$

으

om

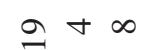

क्त

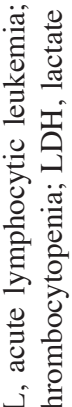

安.

ड़ ठ

造

要

景焉导

额

运

의 을

喑兘

을 昰

纪

플 

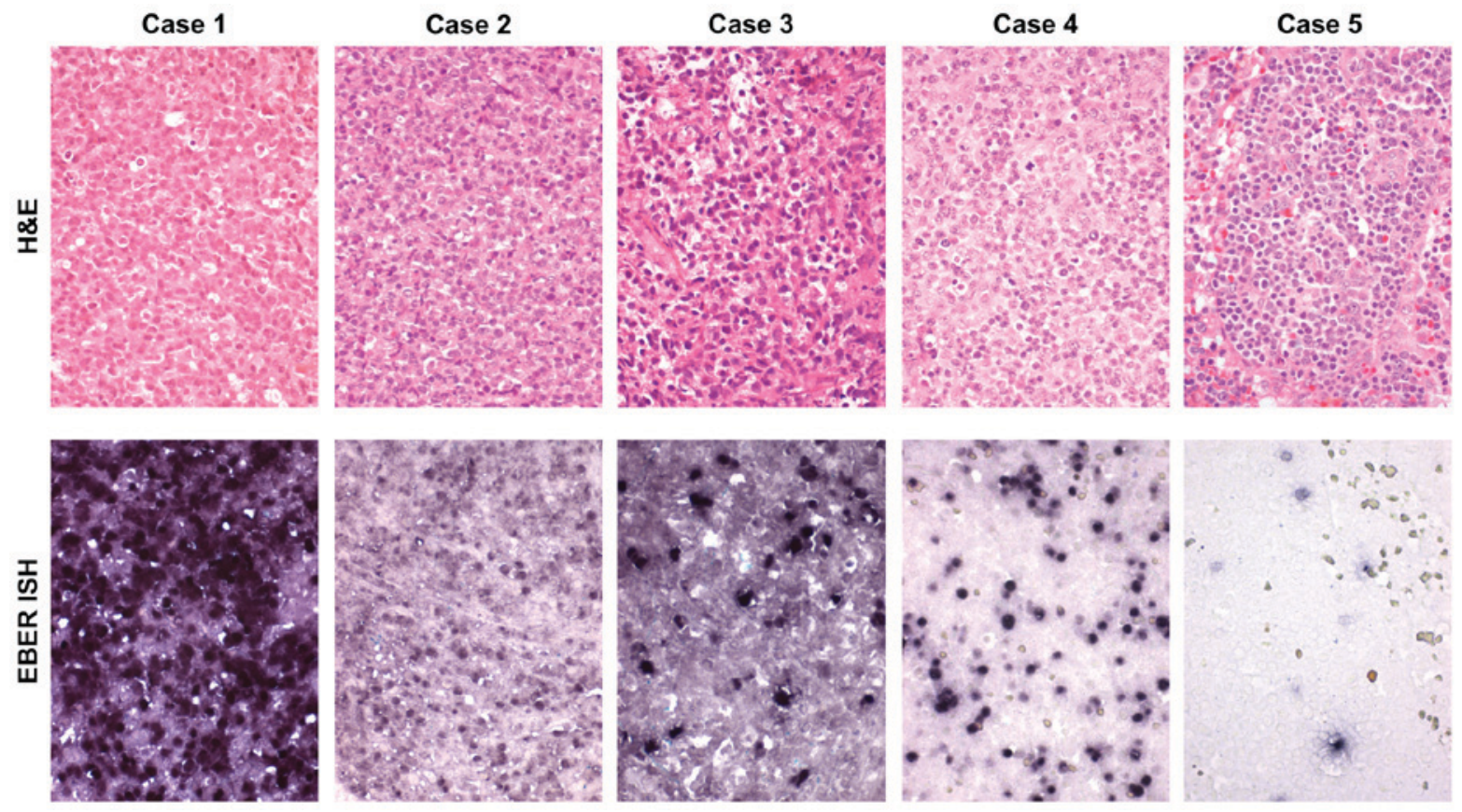

Figure 2. H\&E staining for post-transplant lymphoproliferative disorder diagnosis and classification (magnification, $\mathrm{x} 400$ ). H\&E, hematoxylin and eosin; EBER ISH, Epstein-Barr virus-encoded RNA in situ hybridization.

between 3 weeks to 3 months after transplant (17). Viral infection during this period is correlated with delayed or incomplete reconstitution of specific immunity, or patients experiencing GVHD (18). Regarding PTLD, frequently, the median onset of development is 3 months, with a range of 2-5 months after transplantation (13), which is consistent with our data. Symptoms are quite nonspecific, with patients presenting with fever, malaise, enlarged lymph nodes and high levels of LDH, which were the factors for clinical PTLD suspicion in our patients (2). All patients that developed PTLD had an EBV infection at some point after transplantation. EBV positivity is directly related to PTLD development since its infection, or increase in viral load up to 2,000 copies/ml, occurs mainly at the same time PTLD is diagnosed. PTLD is more frequent in EBV-seronegative patients receiving allografts from $\mathrm{EBV}$-seropositive donors and in patients with delayed immune reconstitution due to T-cell-depletion or HLA-mismatched donor. In a study conducted by Brunstein et al (19), 15 of 335 patients developed a EBV-related complication, at a median of 133 days (range 52-407 days), which is consistent with our results.

As previously described by Bhatia et al (20), PTLD has mortality rates reaching up to $70-90 \%$, which is higher than our results (46.7\%). Survival rates depend on age and stage of disease at the time of diagnosis, with pediatric and patients with localized disease showing the best prognosis (5). In our study, overall patient survival was not affected by the development of PTLD.

This is the first study to describe PTLD cases in HSCT patients in Portugal, combining data from several years at a reference transplantation center. This study demonstrates that EBV infection occurs mainly between 2 and 4 months after transplant and precedes the development of PTLD, and especially the viral load may be important for the monitorization and early diagnosis of PTLD. Thus, the study shows the importance of identify high-risk patients for PTLD development and

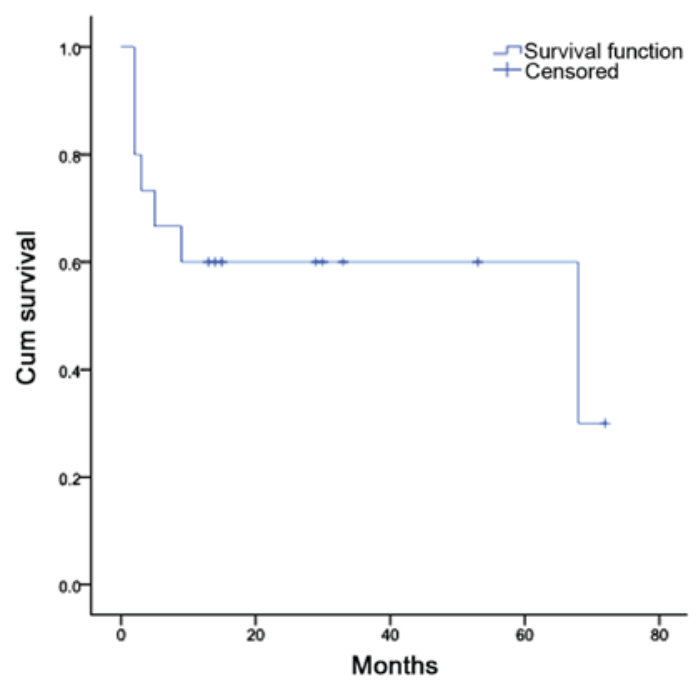

Figure 3. Kaplan-Meier plot for survival analysis of patients with post-transplant lymphoproliferative disorder. Cum, cumulative.

to provide them a frequent monitorization of EBV viral load as suggested by recent guidelines $(21,22)$.

\section{Acknowledgements}

The authors would like to acknowledge the support of Mrs. Rute Silva from the Bone Marrow Transplant Service at IPO Porto (Porto, Portugal) who assisted with the collection of clinicopathological data for the present study.

\section{Funding}

Dr JMD received a grant for the development of the project from the Portuguese League Against Cancer (Liga Portuguesa 
Contra o Cancro-Núcleo Regional do Norte) between April and September 2016.

\section{Availability of data and materials}

The datasets used and/or analyzed during the current study are available from the corresponding author on reasonable request.

\section{Authors' contributions}

JMD and HS designed the study, JL and RH performed the histological analysis of cases, and CPV, LR, RB, FC and ACJr revised the clinical information obtained from patients. IB and RM provided the laboratory data, and JMD and HS performed data analysis and drafted the manuscript. All authors read and revised the manuscript.

\section{Ethics approval and consent to participate}

This retrospective study was approved by The Ethics Committee of Portuguese Oncology Institute of Porto (Porto, Portugal). The need for written informed consent was waived due to the retrospective nature of the study.

\section{Consent for publication}

Not applicable.

\section{Competing interests}

All authors declare that they have no competing interests.

\section{References}

1. Castellano-Sanchez AA, Li S, Qian J, Lagoo A, Weir E and Brat DJ: Primary central nervous system posttransplant lymphoproliferative disorders. Am J Clin Pathol 121: 246-253, 2004.

2. Gulley ML and Tang W: Using Epstein-Barr viral load assays to diagnose, monitor, and prevent posttransplant lymphoproliferative disorder. Clin Microbiol Rev 23: 350-366, 2010.

3. Glotz D, Chapman JR, Dharnidharka VR, Hanto DW, Castro MC, Hirsch HH, Leblond V, Mehta AK, Moulin B, Pagliuca A, et al: The Seville expert workshop for progress in posttransplant lymphoproliferative disorders. Transplantation 94: 784-793, 2012.

4. Mucha K, Foroncewicz B,Ziarkiewicz-Wróblewska B, Krawczyk M, Lerut J and Paczek L: Post-transplant lymphoproliferative disorder in view of the new WHO classification: a more rational approach to a protean disease? Nephrol Dial Transplant 25: 2089-2098, 2010.

5. Kalinova L, Indrakova J and Bachleda P: Post-transplant lymphoproliferative disorder. Biomed Pap Med Fac Univ Palacky Olomouc Czech Repub 153: 251-257, 2009.

6. Atalay A, Gökahmetoğlu S, Durmaz S, Kandemir I, Sağlam D, Kaynar L, Eser B, Cetin M and Kilıç H: Investigation of epstein-barr virus and parvovirus b19 DNA in allogeneic stem cell transplant patients. Turk J Haematol 31: 155-160, 2014.

7. Kim MJ, Kim I, Bae HM, Seo K, Park N, Yoon SS, Park S and Kim BK: Hematopoietic stem cell transplantation after posttransplant lymphoproliferative disorder. J Korean Med Sci 25 : 781-784, 2010.
8. Caillard S, Lelong C, Pessione F and Moulin B; French PTLD Working Group: Post-transplant lymphoproliferative disorders occurring after renal transplantation in adults: Report of 230 cases from the French registry. Am J Transplant 6: 2735-2742, 2006.

9. Funch DP, Walker AM, Schneider G, Ziyadeh NJ and Pescovitz MD: Ganciclovir and acyclovir reduce the risk of post-transplant lymphoproliferative disorder in renal transplant recipients. Am J Transplant 5: 2894-2900, 2005.

10. Allen U and Preiksaitis J; AST Infectious Diseases Community of Practice: Epstein-barr virus and posttransplant lymphoproliferative disorder in solid organ transplant recipients. Am J Transplant 9 (Suppl 4): S87-S96, 2009.

11. Bar-Natan $M$ and Nagler A: Epstein-barr virus-associated post-transplant lymphoproliferative disorder. Isr Med Assoc J 8: 205-207, 2006

12. Grywalska E, Markowicz J, Grabarczyk P, Pasiarski M and Roliński J: Epstein-barr virus-associated lymphoproliferative disorders. Postepy Hig Med Dosw (Online) 67: 481-490, 2013.

13. Luo L, Zhang L, Cai B, Li H, Huang W, Jing Y, Zhu H, Zhao Y, Bo J, Wang Q, et al: Post-transplant lymphoproliferative disease after allogeneic hematopoietic stem cell transplantation: A single-center experience. Ann Transplant 19: 6-12, 2014.

14. Bhatia S: Long-term health impacts of hematopoietic stem cell transplantation inform recommendations for follow-up. Expert Rev Hematol 4: 437-452; quiz 453-454, 2011.

15. Ibrahim HA and Naresh KN: Posttransplant lymphoproliferative disorders. Adv Hematol 2012: 230173, 2012.

16. Choi JH, Park BB, Suh C, Won JH, Lee WS and Shin HJ: Clinical characteristics of monomorphic post-transplant lymphoproliferative disorders. J Korean Med Sci 25: 523-526, 2010.

17. Burns DM, Rana S, Martin E, Nagra S, Ward J, Osman H, Bell AI, Moss P, Russell NH, Craddock CF, et al: Greatly reduced risk of EBV reactivation in rituximab-experienced recipients of alemtuzumab-conditioned allogeneic HSCT. Bone Marrow Transplant 51: 825-832, 2016.

18. Safdar A (ed): Principles and Practice of Cancer Infectious Diseases. In: Current Clinical Oncology. Humana Press, New York, NY, 2011.

19. Brunstein CG, Weisdorf DJ, DeFor T, Barker JN, Tolar J, van Burik JA and Wagner JE: Marked increased risk of Epstein-Barr virus-related complications with the addition of antithymocyte globulin to a nonmyeloablative conditioning prior to unrelated umbilical cord blood transplantation. Blood 108: 2874-2880, 2006.

20. Bhatia S, Ramsay NK, Steinbuch M, Dusenbery KE, Shapiro RS, Weisdorf DJ, Robison LL, Miller JS and Neglia JP: Malignant neoplasms following bone marrow transplantation. Blood 87: 3633-3639, 1996.

21. Styczynski J, Einsele H, Gil L and Ljungman P: Outcome of treatment of Epstein-Barr virus-related post-transplant lymphoproliferative disorder in hematopoietic stem cell recipients: a comprehensive review of reported cases. Transpl Infect Dis 11: 383-392, 2009

22. Styczynski J, van der Velden W, Fox CP, Engelhard D, de la Camara R, Cordonnier C and Ljungman P; Sixth European Conference on Infections in Leukemia, a joint venture of the Infectious Diseases Working Party of the European Society of Blood and Marrow Transplantation (EBMT-IDWP), the Infectious Diseases Group of the European Organization for Research and Treatment of Cancer (EORTC-IDG), the International Immunocompromised Host Society (ICHS) and the European Leukemia Net (ELN): Management of Epstein-Barr Virus infections and post-transplant lymphoproliferative disorders in patients after allogeneic hematopoietic stem cell transplantation: Sixth European Conference on Infections in Leukemia (ECIL-6) guidelines. Haematologica 101: 803-811, 2016. 